\title{
¿Perdidos o salvados? El futuro del trabajo frente a la cuarta Revolución Industrial
}

\section{Lost or saved? The future of work in the face of the fourth Industrial Revolution}

\author{
Lautaro N. Rubbi ${ }^{1} \quad$ Bruna Barlaro Rovati ${ }^{2} \quad$ Axel Petraglia $^{3}$ \\ Universidad Argentina de la Empresa, UADE. Buenos Aires, Argentina. \\ Irubbi@uade.edu.ar \\ bbarlarorovati@uade.edu.ar \\ apetraglia@uade.edu.ar \\ 0000-0003-0025-9744 \\ 0000-0002-9360-8079 \\ 0000-0001-5114-8206
}

Citar como: Rubbi, L., Barlaro Rovati, B. y Petraglia, A. (2020). Perdidos o salvados? El futuro del trabajo frente a la cuarta Revolución Industrial. Desde el Sur 12(1), pp. 307-276.

\section{RESUMEN}

El presente análisis tiene por objetivo indagar sobre el interrogante de los empleos que se perderán y ganarán en el futuro de mediano plazo a causa de la robotización y la automatización de funciones laborales, propias de la cuarta Revolución Industrial. Con este fin, analizamos en profundidad diversos textos académicos, además de políticas públicas de distintos países del mundo y documentos emitidos por compañías multinacionales y grupos gremiales y sindicales, que abordan distintos marcos temporales y espaciales en sus predicciones. Existe amplia variabilidad y polarización de conclusiones entre los autores, que no presentan consenso respecto de si habrá ganancia o pérdida neta de puestos de trabajo en términos absolutos. Sin embargo, se pueden advertir tres consensos básicos: 1) los países y sectores más vulnerables son aquellos que dependen de empleos repetitivos y no creativos; además, se verán afectados fundamentalmente los países emergentes

1 Licenciado en Gobierno y Relaciones Internacionales y en Política y Administración Pública por la Universidad Argentina de la Empresa, UADE. Magíster en Estudios Internacionales y candidato a doctor por la Universidad Torcuato Di Tella, UTDT. Becario Doctoral y docente investigador de UADE-Conicet.

2 Licenciada en Gobierno y Relaciones Internacionales por la Universidad Argentina de la Empresa, UADE, y docente en la misma casa de estudios. Investigadora especialista en Relaciones Internacionales y Seguridad Internacional.

3 Estudiante avanzado de la Licenciatura en Gobierno y Relaciones Internacionales de la UADE. 
o en desarrollo; 2) se espera una elevada fragmentación social con concentración de puestos de trabajo en los dos extremos de la escala de salarios y de exigencias cognitivas; y 3) la educación se presenta, hoy más que nunca, como el factor clave para elevar los niveles de vida, a nivel individual y social.

\section{PALABRAS CLAVE}

Cuarta Revolución Industrial, futuro del empleo, automatización, desigualdad social

\section{ABSTRACT}

This narrative bibliographical analysis aims to inquire into how many jobs will be lost or gained in the mediumterm future as a result of the automation of labor being introduced as part of the fourth Industrial Revolution. The main focus is upon academic texts, coupled with analysis of the public policies of different countries around the world and the business documents of multinational companies, which address different time and spatial frameworks in their predictions. There exists a wide variety of conclusions among different authors, with no consensus on whether there will be an overall gain or loss of jobs in absolute terms; however, we identify three basic consensuses: 1) The most vulnerable countries and sectors are those that depend upon repetitive rather than creative jobs. Emerging or developing countries will be fundamentally affected; 2) A high degree of social fragmentation is expected, with a concentration of jobs at both extremes of the salary and cognitive demands scales; and, 3) More than ever before, education will be the key factor in the raising of living standards, at both individual and societal levels.

\section{KEYWORDS}

Fourth Industrial Revolution, future of employment, automation, social inequality

\section{Introducción}

Nos encontramos ante una época de cambios y un cambio de época. La tendencia hacia la digitalización que Klaus Schwab, fundador y presidente ejecutivo del Foro Económico Mundial (2016), designó como cuarta Revolución Industrial, pone a las empresas, los trabajadores, los Gobiernos y las sociedades frente a un escenario donde se vislumbran retos, 
amenazas y oportunidades, principalmente en el mercado del empleo. El descenso de los costos de las nuevas tecnologías ha creado incentivos para que los empleadores sustituyan la mano de obra humana por capital informático, y ha hecho que muchas fábricas, centros de distribución, tiendas y restaurantes vayan por el camino de la robotización y el uso de la inteligencia artificial. Esto impacta no solo en el trabajo manual (blue collar workers), sino también en el cognitivo (white collar workers).

La rápida disminución en los precios de los robots que se viene dando en los últimos años, inevitablemente, los colocará al alcance de más usuarios. Los robots ya están realizando muchas tareas simples de servicio, como aspirar el polvo, cortar el césped y limpiar alcantarillas. Pero ahora los robots de servicio comercial también pueden realizar tareas más complejas en la preparación de alimentos y cuidado de la salud. Se destacan también los ejemplos de centros de distribución de productos operados enteramente por robots, tiendas sin cajeros, restaurantes totalmente automatizados y asistentes ejecutivos virtuales.

A la par con los avances en robotización, las premisas sobre lo que pueden hacer las computadoras se han expandido recientemente. La informatización se ha limitado históricamente a tareas rutinarias no cognitivas basadas en reglas (Autor, Levy y Murnane, 2003; Goos, Maning y Salomons, 2009; Autor y Dorn, 2013); pero los algoritmos basados en inteligencia artificial y big data ahora están ingresando rápidamente en nuevos dominios y podrían sustituir fácilmente a los humanos en una amplia gama de tareas cognitivas no rutinarias, como solucionar problemas, entender el lenguaje humano, manejar un automóvil o diagnosticar enfermedades (Brynjolfsson y McAfee, 2011; MGl, 2013). Algoritmos sofisticados ya están asumiendo gradualmente una serie de tareas realizadas por secretarias, agentes de viajes, asistentes legales y financieros, abogados de contratos y patentes, y ayudando en tareas de logística y diagnóstico médico. Se espera también que la capacidad de procesamiento de lenguaje natural tenga impacto en servicios públicos como telefonía, banca y salud.

Por supuesto, muchas ocupaciones que se ven afectadas por estos desarrollos aún están lejos de ser totalmente automatizables, lo que significa que la informatización de algunas tareas simplemente liberará tiempo para que el trabajo humano realice otras. No obstante, la tendencia es clara: las computadoras desafían cada vez más el trabajo humano en una amplia gama de tareas cognitivas (Brynjolfsson y McAfee, 2014). En los últimos 10 años, se ha reducido en más del 20\% el trabajo de bibliotecarios, traductores o agentes de viaje. Ingenieros, abogados, contadores y otros profesionales de cuello blanco del sector público y privado no son inmunes a este peligro (Beliz, 2017). 
Frente a estos avances, el futuro del empleo ${ }^{4}$, actualmente, ocupa un lugar central entre las preocupaciones de investigadores, responsables de políticas y empresarios. Cabe enfatizar su inclusión por primera vez en la agenda del G20 de 2018, por petición de la Argentina, lo que da cuenta de la importancia internacional que se le ha asignado al tópico. En segundo lugar, se destaca el aumento en la cantidad de investigaciones de envergadura encargadas por organismos nacionales e internacionales. Estas publicaciones acompañan a una serie de indagaciones académicas también cada vez más numerosa. La amplia variedad de predicciones da cuenta del debate abierto sobre el método adecuado para predecir el riesgo de automatización. Mientras que muchos imaginan un futuro en el que los robots sustituyen a millones de trabajadores en una amplia variedad de tareas, aumenta la desigualdad de ingresos y conduce al desempleo tecnológico y a rupturas en el orden social, otros esperan un futuro en el que la creatividad humana continúe creando nuevos empleos y nuevas formas de cooperación entre los seres humanos y las máquinas (Smith y Anderson, 2014, p. 5).

Ante la gran cantidad (y variabilidad) de predicciones sobre las consecuencias de la automatización en el futuro del empleo, el presente trabajo pretende analizar las conclusiones de diversas fuentes de libre acceso elaboradas en los últimos 10 años, a fin de responder la interrogante sobre cómo se verán afectados cuantitativamente en términos de oferta y demanda los mercados laborales del futuro bajo el impacto de la cuarta Revolución Industrial ${ }^{5}$. El objetivo de este trabajo es identificar, analizar, valorar e interpretar el cuerpo de conocimientos sobre este tema, a fin de evaluar algunas de las conclusiones abordadas, advertir algunas limitaciones y plantear perspectivas propias y futuras líneas de investigación al respecto.

4 Cabe distinguir entre los conceptos de empleo y trabajo, ya que definen situaciones distintas. El primero hace referencia a todas aquellas personas en edad de trabajar que, durante un corto periodo de tiempo, se dedicaron a cualquier actividad para producir bienes o prestar servicios a cambio de un salario o ganancia. Comprende personas empleadas «en el trabajo", es decir, que desempeñaron un trabajo durante al menos una hora; y personas empleadas "que no están en el trabajo» debido a la ausencia temporal de este o por arreglos de horario. En contraste, el trabajo comprende cualquier actividad realizada por personas de cualquier sexo y edad para producir bienes o proporcionar servicios para el uso de terceros o el uso propio. Se define independientemente de su carácter formal o informal, o de la legalidad de la actividad, y puede realizarse en cualquier tipo de unidad económica (Organización Internacional del Trabajo, 2017).

5 Este análisis descarta (por exceder los objetivos de la investigación) aquellas fuentes que se refieren a las consecuencias de otras importantes fuerzas estructurales, como los cambios demográficos, los efectos de la globalización y el cambio climático, o las coyunturas políticas y comerciales. 
Frente a otros trabajos compilatorios sobre la materia, el presente aporte pretende destacarse por la labor de sistematización de los resultados presentados (principalmente en la tabla comparativa, eje central del documento), el abordaje en términos de áreas de consensos y disensos y, más allá de la comparación de resultados entre los diversos documentos, la discusión sobre el avance en la metodología de cálculo y la diversidad de criterios adoptados, dimensión comúnmente desatendida por otros trabajos comparativos, que se limitan a mencionar los resultados de otros estudios. Esta dimensión es fundamental para comprender la razón detrás de la diversidad de predicciones presentadas por distintos especialistas.

La amplia variabilidad de predicciones entre las diversas fuentes abordadas no permite llegar a conclusiones certeras y consensuadas sobre la ganancia o pérdida neta de puestos de trabajo a nivel mundial; sin embargo, cabe destacar que algunas de las predicciones más pesimistas han quedado superadas por las críticas respecto de su metodología de cálculo. Asimismo, se advierten tres consensos básicos en la bibliografía analizada: 1) los países y sectores más vulnerables son aquellos que dependen de empleos repetitivos y no creativos; se verán afectados fundamentalmente los países emergentes o en desarrollo; 2) se espera una elevada fragmentación social con concentración de puestos de trabajo en los dos extremos opuestos de las escalas de salarios y de exigencias cognitivas; y 3) la educación se presenta, hoy más que nunca, como el factor clave para elevar los niveles de vida, a nivel individual y social.

\section{La literatura sobre el futuro del trabajo}

La literatura sobre el futuro del trabajo ha sido prolífica durante los últimos 10 años. Una búsqueda de palabras clave como «futuro del trabajo», «cuarta revolución industrial», «industria 4.0», «empleo y automatización» y "robotización» en algunas de las principales bases de datos académicas (EconLit, Jstor, RePEc, SpringerLink, EBSCO, entre otras) y en buscadores online como Google y Google Scholar permite advertir centenares de documentos que pretenden alcanzar este tipo de predicciones desde diversas metodologías, como regresiones estadísticas, encuestas, entrevistas con empresarios y análisis históricos, entre otras.

Sin embargo, cabe resaltar que el número de estudios de distintas fuentes que plantea predicciones numéricas concretas es, en realidad, muy escaso respecto del total de artículos que discuten los efectos de la automatización. Si bien la literatura sobre el tema es amplia, son pocos los estudios que esbozan pronósticos concretos a partir de metodologías científicamente admisibles. La mayor parte de ellos, si bien discute las posibles consecuencias de los mencionados procesos, evita realizar 
predicciones específicas. Si bien las proyecciones hacia el futuro a partir de modelos matemáticos, percepciones poblacionales a partir de encuestas y otro tipo de metodologías mantienen cierto carácter especulativo, los resultados de este tipo tienen mayor capacidad de ser comparados frente a otros documentos, lo que facilita la sistematización de la bibliografía disponible y la estructura en categorías más claras.

A continuación, se presenta una tabla comparativa con algunos de los principales documentos de libre acceso sobre el futuro del empleo que se han publicado en los últimos 10 años.

6 Si bien se encontraron centenares, optamos por revisar en profundidad 70 documentos provenientes de distintos campos, tales como la sociología, la economía, la ingeniería y la ciencia política (lo que da cuenta del carácter multidisciplinario del objeto abordado). De los mismos, únicamente se extrajeron 30 para su inclusión en la tabla comparativa. Se tomó como principal criterio de selección su calidad en términos metodológicos, su originalidad en términos de aporte novedoso o distintivo y, principalmente, su relevancia en términos del impacto que ha tenido cada documento en la discusión académica sobre el futuro de trabajo (medido fundamentalmente a partir del número de citas y referencias de terceros hacia el mismo). La tabla incorpora únicamente aquellos estudios que incluyen predicciones numéricas concretas sobre la ganancia o pérdida de empleos, y deja fuera una prolífica literatura que aborda los efectos generales de los procesos estructurales analizados desde una perspectiva más bien cualitativa, teórica o formal. Los principales resultados de algunos trabajos dejados de lado se incluyen en la sección posterior, que aborda la discusión sobre el futuro del empleo a causa de la automatización en términos cualitativos. 


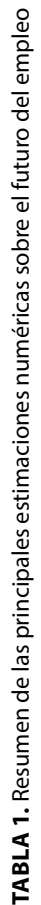
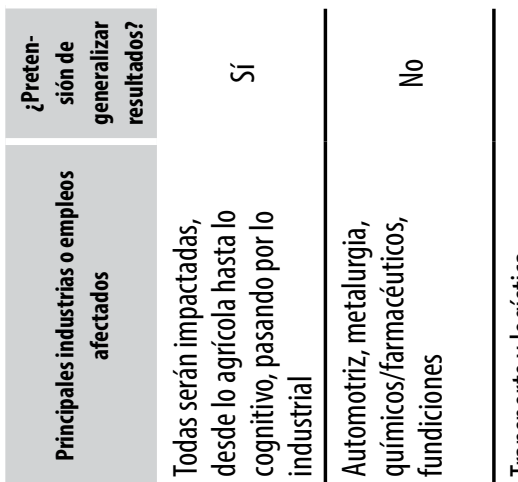

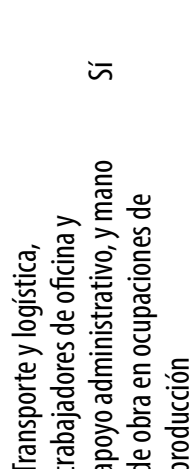
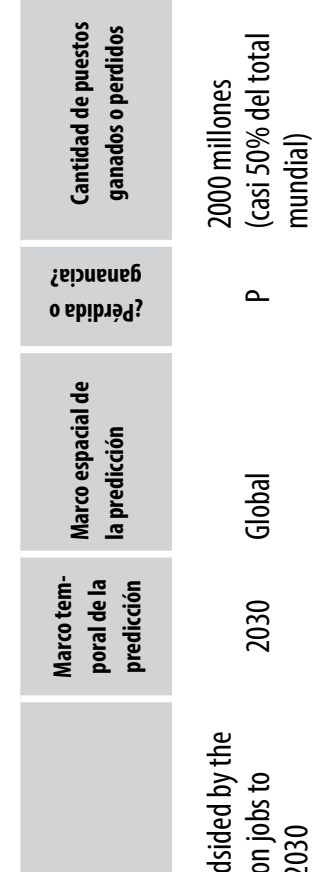

$<0$

\section{Perdidos 0 salvados? El futuro del trabajo trente a la cuarta kevolucion lndustrial}

\begin{tabular}{|c|c|c|c|c|c|}
\hline 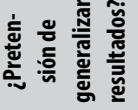 & $i \pi$ & 운 & $i \pi$ & $\stackrel{0}{z}$ & $i \pi$ \\
\hline 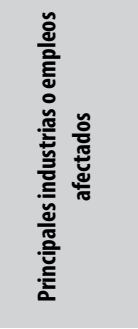 & 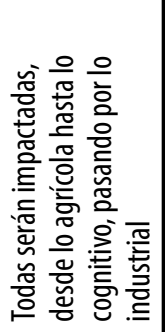 & 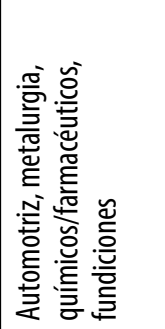 & 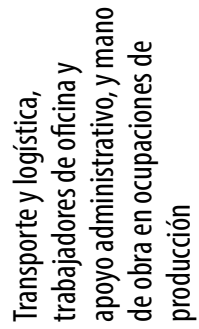 & 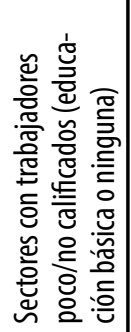 & 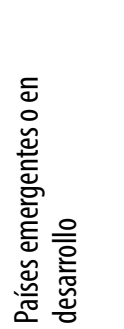 \\
\hline 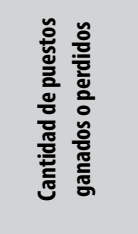 & 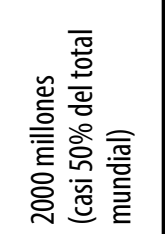 & 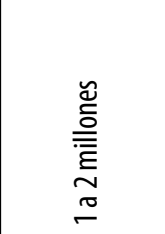 & 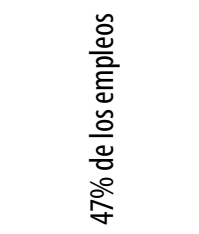 & 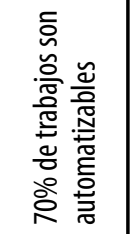 & 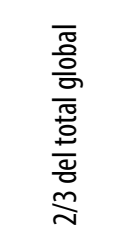 \\
\hline 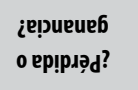 & $a$ & ৩ & a & $a$ & $a$ \\
\hline 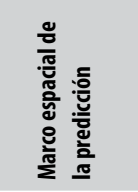 & $\begin{array}{l}\overline{\widetilde{0}} \\
\text { 은 }\end{array}$ & 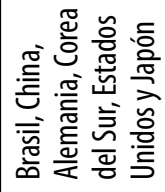 & 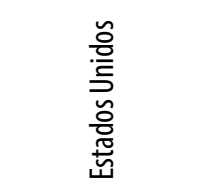 & 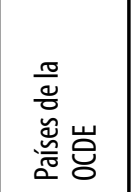 & $\begin{array}{l}\text { 즘 } \\
\text { 吾 } \\
\text { 产 }\end{array}$ \\
\hline 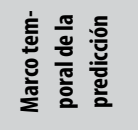 & ஜ̊ & 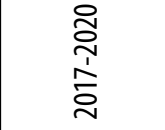 & 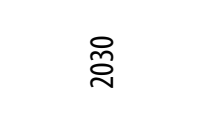 & ' & 움 \\
\hline$\stackrel{\circ}{\underline{E}}$ & 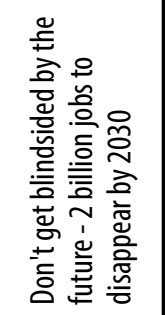 & 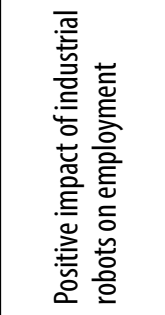 & 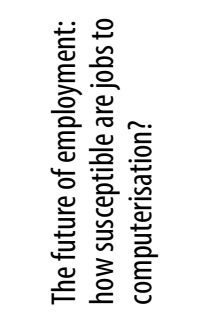 & 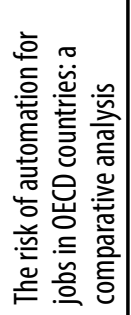 & 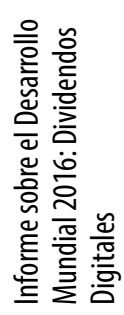 \\
\hline ouy & rlor & $\varepsilon 10 Z$ & દLOZ & 9102 & 9102 \\
\hline 旁 & 岕 & 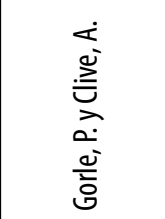 & 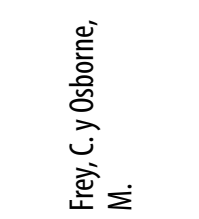 & 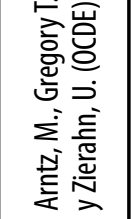 & 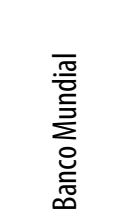 \\
\hline
\end{tabular}




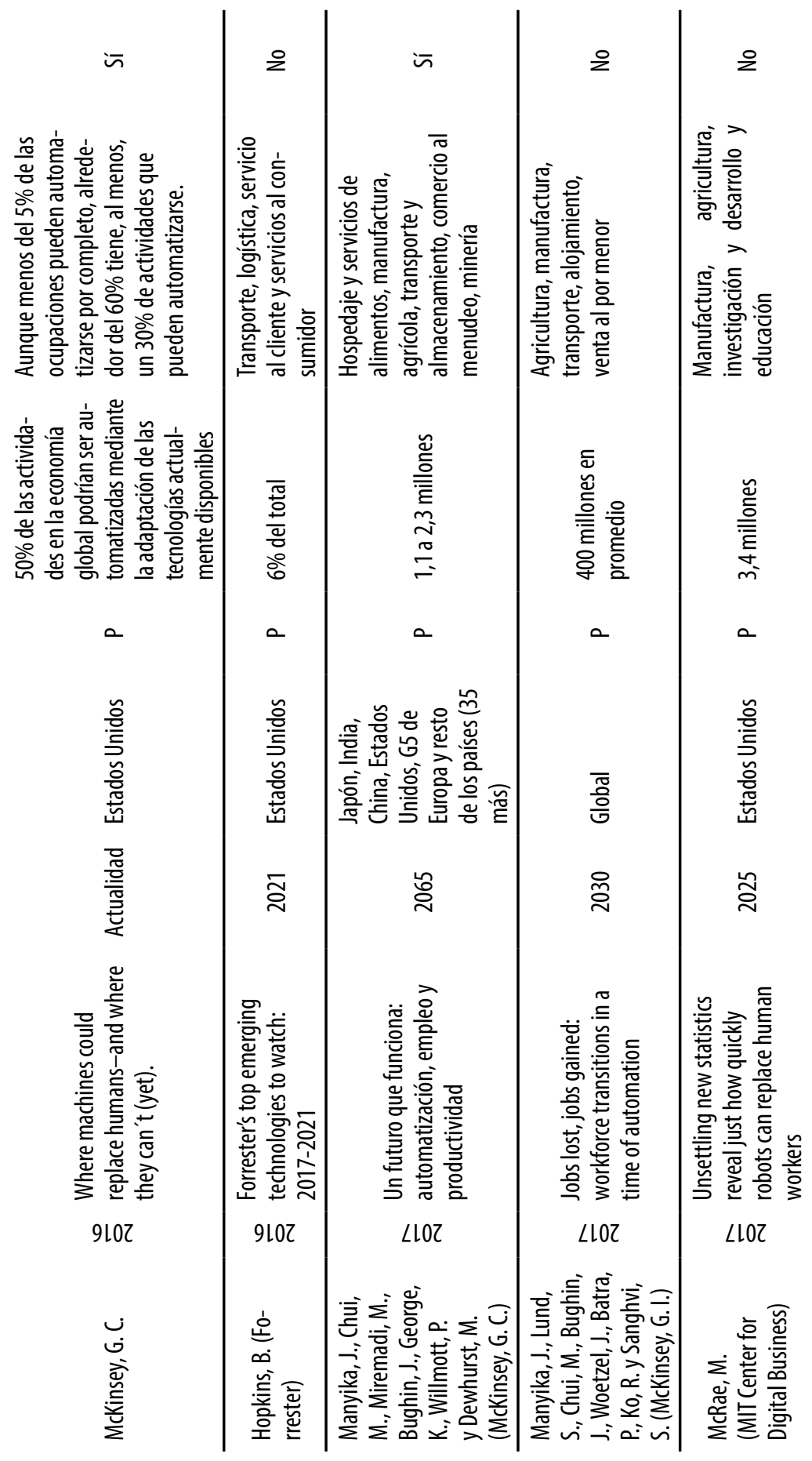




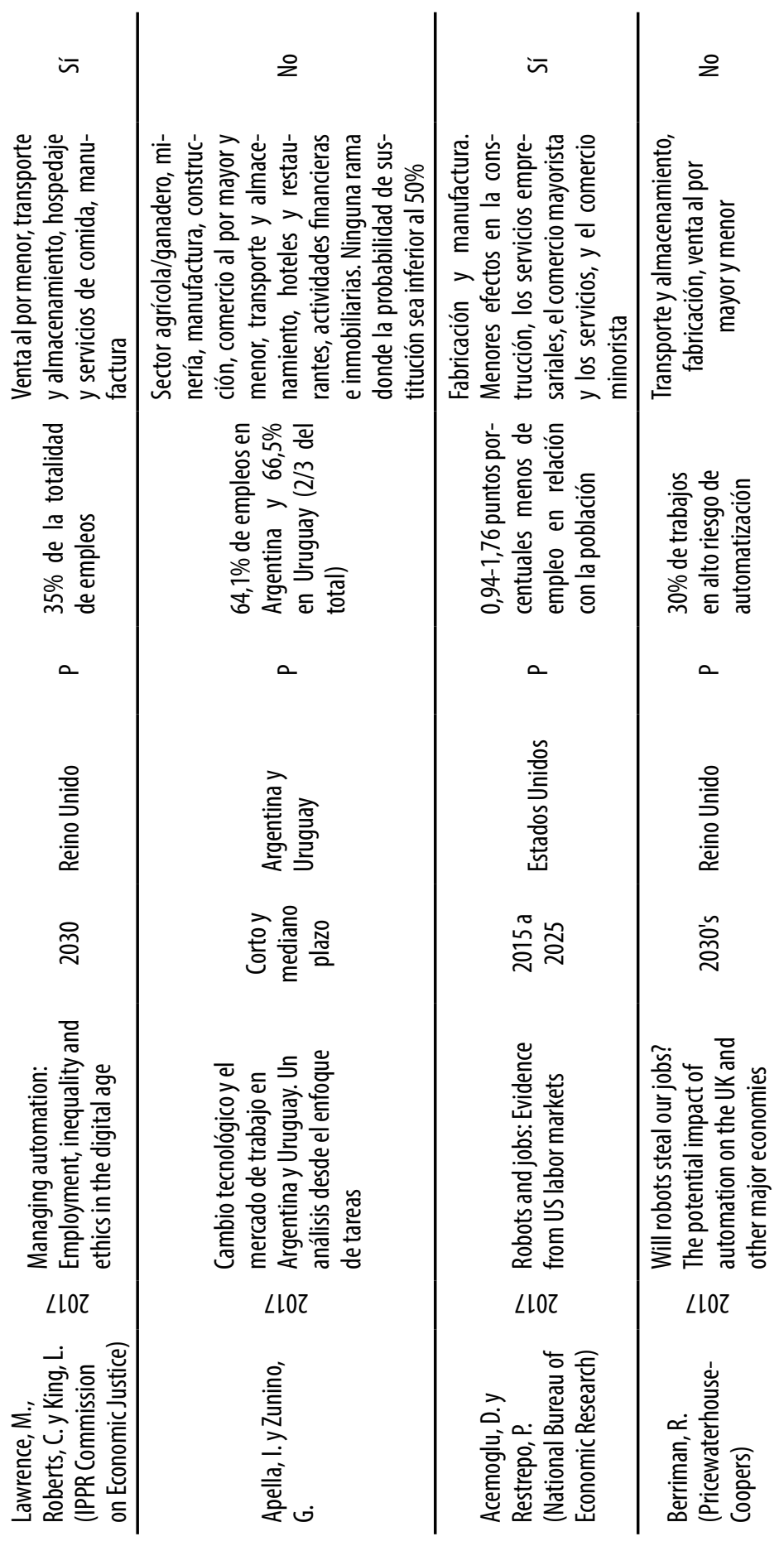




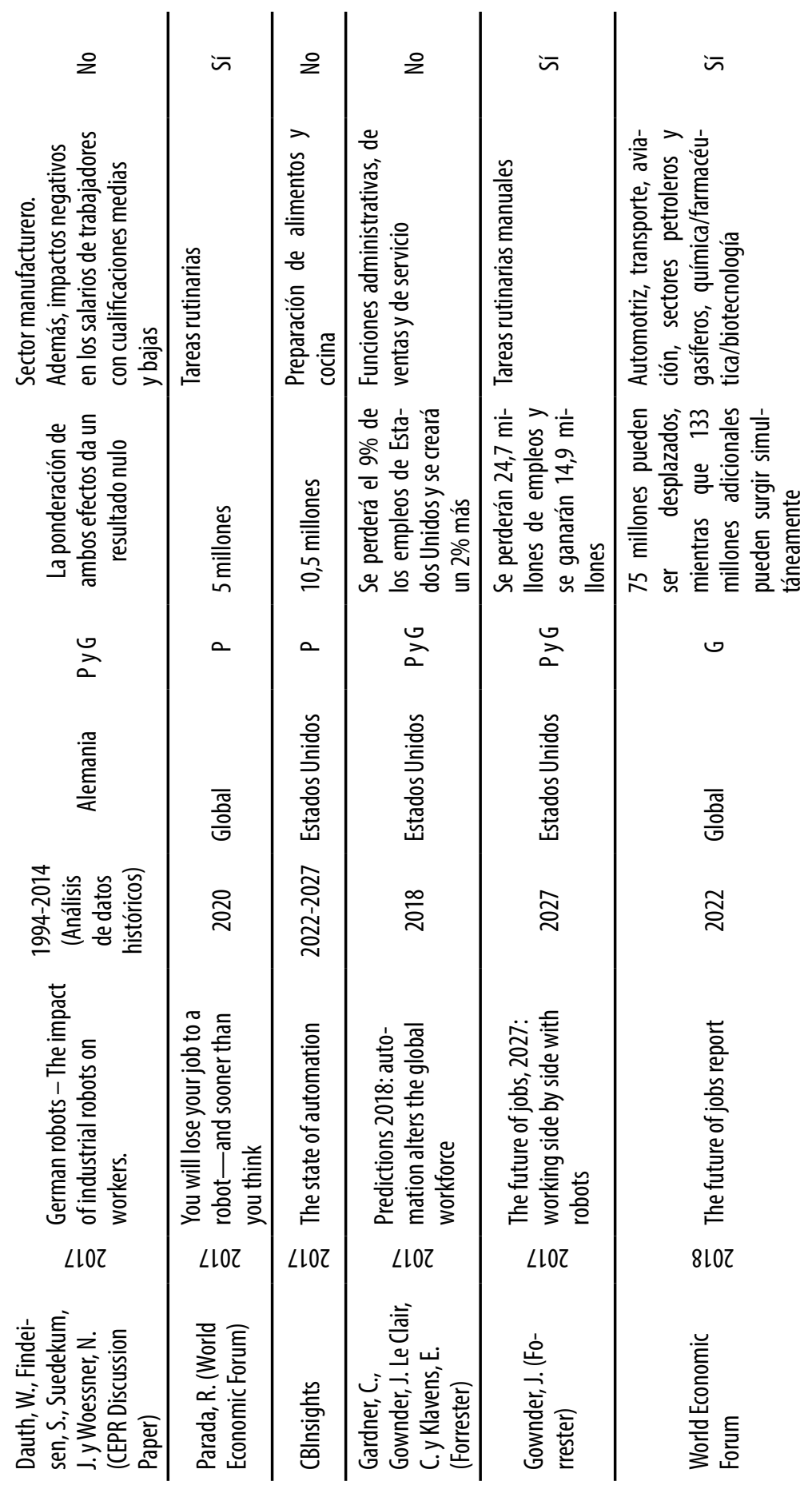




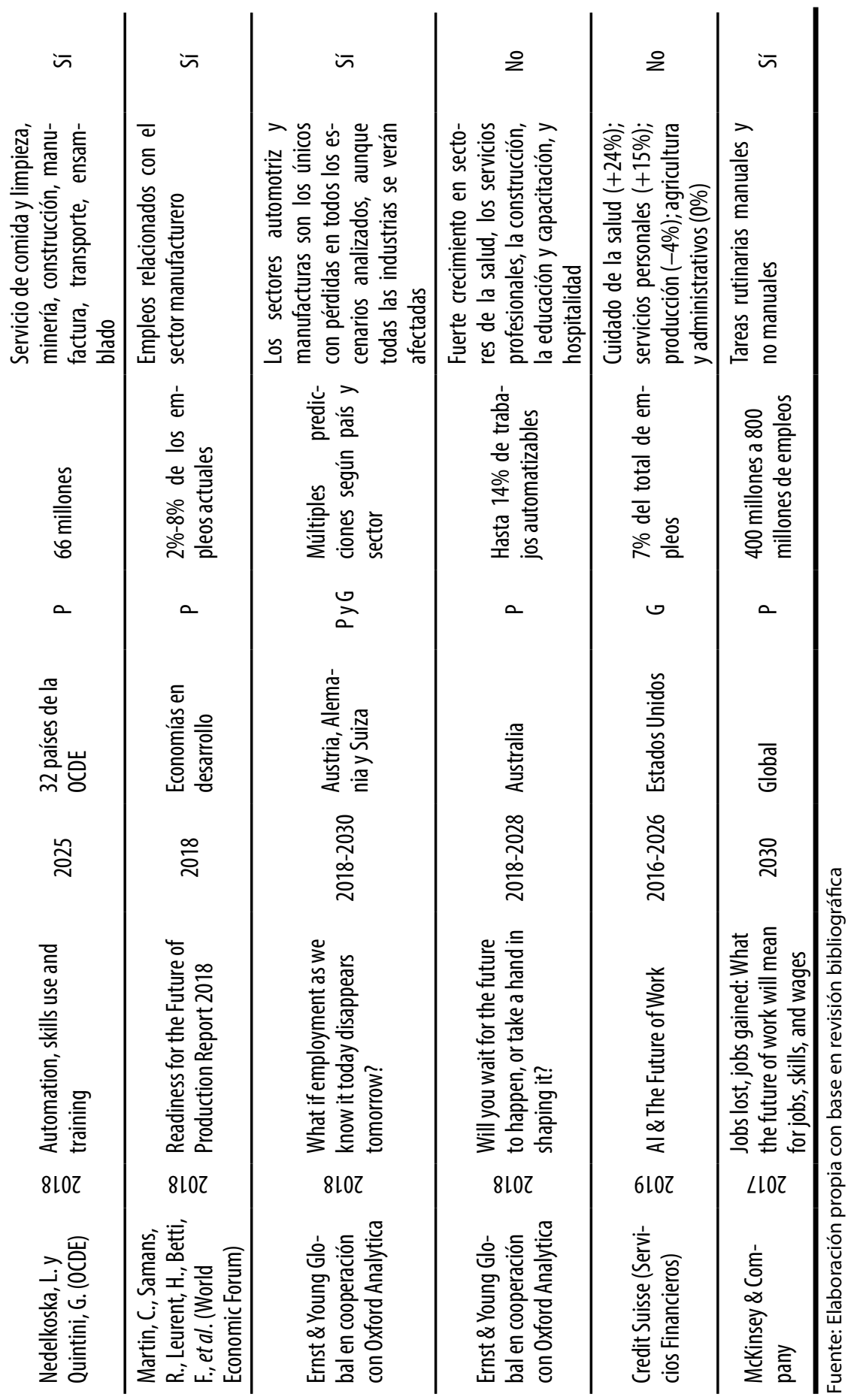


Académicos, grupos de reflexión y responsables políticos han alimentado ricos debates sobre cómo podría ser el futuro del empleo y la manera de darle forma. Sin embargo, a pesar de la creciente investigación en este campo, no existe una definición universalmente aceptada sobre qué abarca exactamente el «futuro del trabajo» y cuáles son los factores más relevantes. En consecuencia, existe una gran variedad de temas y métodos cubiertos por la literatura que apenas encuentra algunos consensos básicos.

En este sentido, los cálculos estadísticos sobre la ganancia o pérdida neta de empleos son solo una de las aristas de la temática del futuro del empleo. La literatura cubre también un amplio espectro que va desde la calidad de estos trabajos hasta la sustentabilidad de los programas de protección social, y pasa por las relaciones entre empresas y sindicatos, las cuestiones de desigualdad social, el impacto sobre los salarios de los trabajadores remanentes y el futuro de la educación para afrontar estos cambios, entre muchas otras cuestiones.

Asimismo, es importante destacar que la automatización, principal fuerza estructural que se considera en esta investigación, no es la única fuerza que impactará sobre el futuro del trabajo. Otros factores importantes por considerar son los cambios demográficos, marcados tanto por las tasas de envejecimiento, natalidad y mortalidad como por el fenómeno creciente de las migraciones; los cambios en materia de educación; el cambio climático; los cambios en los patrones de consumo y las lógicas propias de los contextos políticos y económicos coyunturales a nivel nacional, regional e internacional.

En esencia, pueden advertirse dos grandes grupos con opiniones divergentes sobre las consecuencias de los procesos de automatización, que se presentan como los dos extremos de un continuo con diversos grises de por medio.

\section{Los pesimistas tecnológicos}

En primer lugar, autores como Frey y Osborne (2013) y Apella y Zunino (2017) entienden que la automatización plantea consecuencias fuertemente negativas para el mercado laboral, principalmente desde el planteo del inminente reemplazo de los trabajadores que ejecutan tareas manuales rutinarias por la robótica y la potencial sustitución de aquellos que realizan tareas cognitivas rutinarias por la inteligencia artificial.

Dentro de este grupo, que podríamos denominar como los «pesimistas tecnológicos», la mayoría de los observadores parecen estar de acuerdo en que es probable que la destrucción de puestos de trabajo se acelere bajo los cambios actuales (Frey y Osborne, 2013; Arntz, Gregory y Zierahn, 
2016; Decanio, 2016; Manyika et al., 2017). Por el contrario, mantienen que se sabe poco sobre el potencial de creación de nuevos puestos de trabajo. Para que aparezcan estos nuevos puestos, muchos comentan la necesidad de desarrollar y regular nuevos mercados, en particular en los sectores de la economía verde, la asistencia y los servicios personales, o de aumentar el sector público en áreas en las que actualmente no existen actividades rentables (Pollin et al., 2014; ILO, 2017a). El temor es que este proceso no se lleve a cabo con la suficiente rapidez. Por lo tanto, el número de puestos de trabajo podría disminuir más rápidamente que el de la mano de obra mundial cuando los puestos de trabajo existentes se sustituyan por la automatización y otros sistemas operados por la inteligencia artificial.

El trabajo de Frey y Osborne es uno de los más influyentes y citados dentro de esta corriente, en tanto fueron los primeros investigadores en intentar cuantificar el impacto de la tecnología en una amplia gama de industrias y ocupaciones dentro de un país específico (Estados Unidos) utilizando modelos predictivos. Su modelización mostró que hasta un $47 \%$ de los puestos de trabajo dentro de Estados Unidos estaban en riesgo de desaparecer debido al impacto de la automatización.

Como resumen de Valenduc y Vendramin (2017), la metodología de Osborne y Frey se ha aplicado para pronosticar las probabilidades europeas de sustitución de puestos de trabajo en distintos estudios (RolandBerger, 2014; Bowles, 2014; Deloitte, 2014, 2015; Baert y Ledent, 2015), y se han encontrado probabilidades similares de automatización (del 30\% al 40\%). A partir de la misma metodología, las estimaciones indican que alrededor del $44 \%$ de los puestos de trabajo australianos corrían peligro de automatización para 2030 (Edmonds y Bradley, 2015). Chui, Manyika y Miremadi (2019), por su parte, estiman que, a nivel mundial, la automatización afectará a 1100 millones de trabajadores (49\% de los puestos de trabajo) y US\$ 12700 millones en salarios. Un informe del Banco Mundial (2016) evalúa que, para 2040, dos tercios de la totalidad de los empleos en los países en desarrollo será afectada por la automatización.

En un reconocido trabajo, Acemoglu y Restrepo (2017) examinan el impacto de introducir robots en la producción en Estados Unidos. Sus resultados no son alentadores: su veredicto es que en los últimos años la incorporación de los robots industriales tuvo impactos negativos en la demanda de trabajo y los salarios. En este caso, los efectos de pérdida de empleos superan los efectos positivos causados por el abaratamiento de la producción de bienes y servicios. Por ahora, la pérdida neta de empleo ha sido pequeña, ya que la prevalencia de robots, si bien ha crecido mucho, es todavía baja. Sin embargo, la robotización crecerá de forma importante en los próximos años. Para reforzar esta idea, un estudio de la OCDE 
del mismo año advierte que solo el $13 \%$ de los trabajadores utilizan sus habilidades cognitivas y de resolución de problemas cotidianamente con niveles de rendimiento superior a los que actualmente pueden conseguir las computadoras, mientras que dos tercios los utilizan al mismo nivel que estas, por lo que pueden ser reemplazados sin grandes costos en el futuro cercano (OCDE, 2017).

En un estudio Delphi realizado por la Fundación Bertelsmann (2015), con resultados ligeramente menos dramáticos, aunque igual de aflictivos, los expertos prevén que el desempleo aumentará continuamente tanto en las economías avanzadas como en las emergentes, hasta alcanzar más del 20\% en Europa y más del 25\% en América Latina y del Norte para 2050.

Igualmente, cabe destacar que, aunque muchos de los titulares más alarmantes reflejan preocupaciones sobre si una ocupación específica podría automatizarse, pocos consideran si efectivamente lo será. Incluso si consideramos que estas estimaciones son correctas en términos de factibilidad tecnológica, que exista la posibilidad de automatizar un trabajo no implica necesariamente que sea económicamente viable hacerlo. En este sentido, el plazo para adaptarse quizás pueda ser algo mayor al que a priori se supone. Mientras que diversos analistas proyectan sus predicciones hacia los próximos 15 o 20 años, Manyika et al. (2017), por ejemplo, apoyan una hipótesis de más largo plazo, que tiene en cuenta tanto los costes de aplicación y las contingencias del marco reglamentario como los principales factores que influirán en el ritmo y el alcance del proceso de adopción de las nuevas tecnologías. Sus estimaciones sugieren que el reemplazo de trabajadores por procesos automatizados ocurrirá con mayor seguridad hacia 2055, aunque también podría ocurrir 20 años antes o después, lo cual depende de estos factores y del hecho de que el potencial de susceptibilidad a la automatización varía entre ocupaciones (Manyika et al., 2017, pp. 5-6).

En línea con esta dimensión de análisis, la famosa revista The Economist (2018) lanzó el índice de preparación para la automatización. El hallazgo clave es que ninguna nación incluida en el estudio está completamente preparada para enfrentar el desafío. Un puñado de países con una sólida educación, formación de trabajadores y sectores de investigación y desarrollo, como Corea del Sur, Alemania y Singapur, tienen importantes ventajas. Pero incluso ellos, junto con el resto del mundo, necesitarán tomar medidas audaces para prepararse ante la próxima ola de automatización. Por su parte, el Foro Económico Mundial lanzó el mismo año un índice similar. La evaluación mide la preparación para el futuro de la producción teniendo en cuenta la estructura de producción y otros factores clave que posicionan a un país para capitalizar la cuarta Revolución Industrial. 
Los resultados señalan la probable polarización de la producción en un mundo a dos velocidades. De las 100 economías incluidas, solo 25 países de Europa, América del Norte y Asia oriental son países líderes o están en las mejores condiciones para beneficiarse de la naturaleza cambiante de la producción. Cerca del $90 \%$ de los países de América Latina, Medio Oriente, África y Eurasia incluidos en la evaluación se clasifican como países nacientes, el grupo menos preparado para el futuro de la producción (WEF, 2018). Puede advertirse, en este sentido, que las decisiones políticas y los diversos modelos de tratamiento político y normativo del trabajo y el empleo, junto con las políticas públicas en términos de educación, serán variables fundamentales que moderarán el impacto de las nuevas tecnologías sobre la dimensión laboral.

Al respecto, algunos autores toman el análisis de los diferentes modelos políticos existentes en torno al desenvolvimiento de la automatización del trabajo. Atkinson y Brown (2019) parten del presupuesto de que la nueva ola tecnológica afectará a los mercados laborales nacionales de formas similares, al menos en los países desarrollados con estructuras industriales y niveles salariales semejantes. Sin embargo, las economías nacionales se verán afectadas de país a país, considerando que tales variaciones se deben a distintos factores: el riesgo de pérdida de trabajos automatizables, la estructura económica, las condiciones sociales, las condiciones del mercado laboral, los apoyos brindados al mercado laboral, las capacidades del trabajador y los incentivos y límites de despido (Atkinson y Brown, 2019, p. 51). En un estudio comparativo realizado por estos autores tomando los casos de Estados Unidos, Francia, España y Alemania, estiman que es este último país ${ }^{8}$ el que estaría mejor posicionado para afrontar los cambios derivados de la tecnología y la automatización, porque destaca positivamente en las dimensiones de condiciones sociales, capacidades de los trabajadores e incentivos y límites a los despidos (Atkinson y Brown, 2019, p. 57).

\footnotetext{
8 Alemania representa un caso exitoso por varias razones, entre ellas que fue el primer país en regular de forma federal la adopción de la llamada Industria 4.0 (robótica, inteligencia artificial e IOT aplicadas a la producción industrial avanzada), al definir las «tres leyes de la robótica" que toma normas éticas por aplicar en los sectores de esta industria. Asimismo, implementó un sistema de educación dual por el cual los trabajadores de 1 de cada 5 empresas alemanas son capacitados en torno a los cambios tecnológicos adoptados para mantener un personal altamente calificado; adoptó un sistema de prácticas laborales muy flexibles que incluye contratos a plazo fijo, sistemas de turnos y permisos operativos 24/7; lanzó un programa de incentivos públicos y programas de investigación y desarrollo para diversos rubros de empresas de la industria 4.0; y, por último, promocionó el ingreso a una serie de universidades especializadas en ingeniería mecánica con una diversidad de programas de estudio de robótica y automatización (Germany Trade \& Invest, 2018).
} 
En un plano más amplio, Torres Muñoz (2019) aborda las limitaciones en otros casos de países desarrollados y explica que, a nivel de Unión Europa, hoy en día, no se han establecido regulaciones de estandarización del uso de la robótica. El debate se centra todavía en el mundo académi$\mathrm{co}$, con una literatura relativamente abundante, pero sin llegar a grandes acuerdos que normen el uso de los robots y garanticen una transición medianamente favorable hacia la automatización. Para el caso de Estados Unidos, sí se han hecho avances en cuanto a las regulaciones de la robótica y la inteligencia artificial. No obstante, las agencias están diseminadas a nivel federal y estatal, y en algunas circunstancias colisionan entre sí, ya sea por inconsistencias, brecha entre ambas legislaciones o una carencia de regulación por parte del ámbito federal (Torres Muñoz, 2019, pp. 4-6).

En el caso de América Latina, el retraso en la adopción de estas tecnologías podría explicarse por una situación de relativo atraso en el desarrollo económico, lo cual ocasionaría que, aun cuando nuevas tecnologías están disponibles para reemplazar tareas rutinarias, el costo relativo de incorporarlas a través de inversiones de capital versus el costo laboral de quienes actualmente las desarrollan no justifique su adopción. En otras palabras, dado un costo relativo del trabajo suficientemente bajo, no se justifica su reemplazo por tecnología en el corto plazo. Este costo dependerá, a su vez, del nivel de los salarios, las cargas sociales, el costo de la energía y la tasa a la que las nuevas tecnologías se deprecien (Galiani, 2017). Otra explicación posible son las barreras de tipo institucional para la incorporación de estas tecnologías, como las restricciones a la importación de bienes de capital y la existencia de acuerdos laborales que garantizan el empleo a quienes podrían ser reemplazados (Rofman y Apella, 2017).

Sin embargo, es esperable que las nuevas tecnologías continúen reduciendo su costo y aumentando su impacto, debilitando la restricción por precios relativos. En el caso de la Argentina, el alto costo de mantener a los trabajadores debido a las cuantiosas cargas sociales que los empleadores deben afrontar los convierte en un objetivo aún más jugoso para los procesos de automatización. Al mismo tiempo, otras restricciones institucionales se harán gradualmente más costosas, en términos económicos y sociales. Estos efectos combinados podrían resultar en una adopción tardía, pero que ofrecería menos oportunidades de adaptación a los trabajadores. En respuesta, las reformas y los progresos en términos de educación en estos países se han tornado más urgentes que nunca, en tanto corren el riesgo de convivir con una población no solo desempleada, sino también no empleable.

Además, algunos observadores temen que los cambios tecnológicos actuales tengan un menor potencial de creación de empleo que otras 
revoluciones del pasado. Oxford Martin School (2015) demuestra que la economía de Estados Unidos ha creado solo el 0,5\% de los puestos de trabajo actuales desde 2000, en comparación con el $8 \%$ durante la década de 1980. Sin embargo, la cuarta Revolución Industrial puede desafiar las tendencias históricas debido al ritmo del progreso tecnológico y a que las tecnologías asociadas son capaces de ejecutar tanto tareas físicas como cognitivas (Schwab, 2016; World Economic Forum, 2017; Estevadeordal et al., 2017). Además, tal como advierte Bessen (2019), «la automatización puede no causar desempleo masivo, pero puede requerir que los trabajadores hagan transiciones perturbadoras a nuevas industrias, requiriendo nuevas habilidades y ocupaciones". Es probable que no todos los países, sectores o individuos estén preparados para afrontar estos cambios, lo que requiere medidas drásticas a nivel de políticas públicas de adaptación.

\section{Los optimistas tecnológicos}

En el otro extremo del continuo antes mencionado, otros académicos como Santanatoglia, Sosa Valle, Mariani y Pivetta (2018), Autor (2015, 2016, 2017) y Rofman y Apella (2017, p. 24) asumen una visión más optimista, bajo dos argumentos principales: por un lado, resaltan la creciente emergencia y demanda de nuevos puestos de trabajo que se generan a partir de los cambios tecnológicos y, por otro, destacan que el supuesto de automatizar un grupo de tareas no elimina la necesidad de otras no automatizadas, por lo cual el escenario apocalíptico del fin del trabajo ejecutado por humanos quedaría descartado.

Respecto de la primera línea, diversos autores argumentan que mientras algunas ocupaciones se desvanecen, otras nuevas nacen, lo que crea aún más empleos de los que se destruyen. La experiencia histórica muestra que el cambio tecnológico es un proceso evolutivo complejo, no lineal y costoso, que desencadena un proceso dinámico de destrucción creativa (Schumpeter, 1942): los nuevos paradigmas tecnológicos vienen en oleadas que tienen diferentes fases y este proceso destruye puestos de trabajo en la primera fase, pero también crea nuevos trabajos en las fases subsiguientes. Esto sucede porque más compañías ingresan a industrias en las que la demanda es relativamente alta, lo que expande el empleo en ellas. Además, un aumento en la eficiencia de la producción que reduce el precio de los bienes aumenta el ingreso real de la sociedad y, por lo tanto, permite el aumento de la demanda de otros bienes. De hecho, históricamente, los avances tecnológicos han tenido efectos netos positivos en el empleo, a medida que los ajustes se movilizan y los procesos se transforman (Atkinson y Wu, 2017). Sin embargo, cabe resaltar que diversos autores coinciden en que, si bien habrá un aumento en la demanda de trabajo, 
este seguramente se concentrará en empleos de alta calificación, difíciles de acceder para la mayor parte de la población (Engler et al. 2018).

Moretti (2010), por ejemplo, argumenta que cada nuevo trabajo en el sector de la tecnología creará aproximadamente 4,9 puestos de trabajo adicionales en el sector de servicios. Esto también fue confirmado por el trabajo de Goos et al. (2015), que presenta hallazgos similares utilizando un conjunto de datos europeos. Por otra parte, en 2017, investigadores del Instituto de Tecnología de Massachusetts y la Universidad de Boston demostraron que la mitad del crecimiento del empleo en Estados Unidos entre 1980 y 2007 provino de la creación y expansión de nuevas categorías laborales. Y algunas evidencias apuntan a que el empleo en realidad creció más rápido en los campos que se estaban computarizando rápidamente.

Los empleos tecnológicos juegan un papel crítico en la creación de demanda para las ocupaciones de servicios locales. Más concretamente, si bien el empleo en la industria manufacturera tiende a disminuir en respuesta a la automatización, estos efectos se ven compensados con creces por el crecimiento del empleo en el sector de los servicios. Diversos estudios estiman que la tecnología podría aumentar las oportunidades de empleo en nuevas ocupaciones, especialmente en arquitectura, ingeniería, informática y matemáticas (Smith y Anderson, 2014; Arntz et al., 2016; World Economic Forum, 2016a; German Federal Ministry of Labour and Social Affairs, 2016; Credit Suisse, 2019).

Según el Foro Económico Mundial (FME), incluso si la robótica comenzara a desplazar a un gran número de trabajadores, los trabajos que dependen de los rasgos humanos, tales como la creatividad y la inteligencia emocional, pueden volverse más numerosos. En Francia, por ejemplo, internet destruyó 0,5 millones de puestos de trabajo en los últimos 15 años, pero también creó 1,2 millones de nuevos puestos, un efecto neto de 2,4 puestos creados por cada puesto destruido (McKinsey, 2016). De hecho, también se espera que estos efectos sean pronunciados en el sector manufacturero situado en el mundo en desarrollo. Un estudio realizado por Berger, Chen y Frey (2016) ha demostrado que el efecto multiplicador asociado con los empleos de manufactura calificada es de 6 a 9 veces mayor en el mundo en desarrollo que en los Estados Unidos.

Luksha et al. (2015) intentan especular sobre las futuras industrias y ocupaciones que podrían surgir dadas las tecnologías actuales. Más concretamente, los autores llevan a cabo un análisis de escenarios a nivel de la industria que investiga los efectos de la tecnología en los puestos de trabajo rusos hasta 2030. Según su punto de vista, si bien es cierto que varios empleos quedarán obsoletos en un futuro próximo, el potencial 
de creación de puestos de trabajo supera a estos despidos, aunque los autores hacen hincapié en la necesidad de que los futuros empleados desarrollen habilidades interprofesionales para seguir siendo competitivos.

Como ejemplos concretos, trabajos como desarrollador de aplicaciones, administrador de redes sociales y operador de drones no existían hasta hace poco; hoy, millones de trabajadores tienen tales cargos. La ocupación del desarrollador web no existía hasta principios de la década de 1990, y durante el 2018 empleó a 150000 estadounidenses con un salario promedio de US\$ 66 000. Asimismo, según Linkedln, los puestos de científicos de datos en Estados Unidos aumentaron un 650\% entre 2012 y 2017. Los salarios anuales medios de estas ocupaciones suelen ser mucho mayores que las ocupaciones que requieren solo habilidades básicas (Laviña Orueta, Serrano y Varela Ferrio, 2019, p. 84).

Al mismo tiempo, a pesar de los temores de que los humanos pierdan frente a las máquinas, hay muchos casos recientes en los que la gente ha trabajado con máquinas y, en el proceso, se ha vuelto más productiva y mejor compensada. El ejemplo más conocido de este fenómeno proviene de la banca. A medida que proliferaron los cajeros automáticos, los empleados de los bancos simplemente cambiaron su enfoque hacia el servicio al cliente. El puesto de cajero se convirtió en una posición de mayor calificación.

Por otra parte, los «optimistas tecnológicos» apuntan contra las mediciones llevadas a cabo por los pesimistas en términos de la metodología que utilizan para predecir la extinción de diversos puestos laborales. En respuesta al frecuentemente citado trabajo de Frey y Osborne, se considera que su metodología produjo una sobreestimación significativa del impacto de los mencionados procesos al asumir que todas las ocupaciones específicas eran las mismas. Frey y Osborne asumieron que, si una ocupación puede ser computarizada, entonces todos los empleos en esa ocupación serían destruidos. Esta es una visión más bien reduccionista, ya que, en la mayoría de los casos, si la tecnología sustituyera a la mano de obra, es poco probable que se pierdan todos los puestos de trabajo de esa familia respectiva. En realidad, advierten los críticos, las ocupaciones consisten en realidad en tareas y la agrupación de tareas dentro de las distintas ocupaciones puede ser muy diferente, y se mantienen en la mayor parte de los casos diversas tareas difícilmente automatizables. Como resultado, la mayor parte de las ocupaciones son bastante menos susceptibles a la informatización de lo que Frey y Osborne esperaban (Autor, 2015; Arntz et al., 2016; alphaßeta, 2017; Bakhshi et al., 2017; OCDE, 2017; Bakhshi, Downing, Osborne y Schneider, 2017; Nedelkoska y Quintini, 2018). 
Más aún, autores como Downey (2016) argumentan que la automatización reduce la complejidad de las tareas, lo cual permite que trabajadores menos calificados realicen trabajos anteriormente impensados. Las conclusiones de Bakshshi et al. (2017), por ejemplo, sugieren que, para aproximadamente el $70 \%$ de las ocupaciones, el rediseño de los puestos de trabajo junto con la reconversión de la fuerza de trabajo tiene el potencial de promover el crecimiento y su propia demanda. Debido a que Osborne es un autor colaborador en este trabajo, el informe tiene un significado adicional, ya que representa resultados revisados basados en una variación del modelo original. Por su parte, el informe de la consultora australiana alphaßeta (asociada a Google), de 2017, concluyó que la tecnología tiene el potencial de aumentar la cantidad de tiempo que los trabajadores pueden dedicar a tareas que son "exclusivamente humanas", lo que aumentaría su productividad e impulsaría la economía australiana en hasta US\$ 2,2 billones para 2030.

En línea con este pensamiento, Autor $(2015 ; 2017)$ revisó sus previsiones pesimistas de su trabajo de 2013 al observar que algunas tareas de carácter manual o abstracto implican reglas tácitas que son difíciles de traducir en un conjunto de reglas explícitas. Según Autor (2015), los periodistas e incluso los comentaristas expertos tienden a exagerar el grado de sustitución de la mano de obra humana por la maquinaria e ignoran las fuertes complementariedades entre la automatización y el trabajo que aumentan la productividad, los ingresos y la demanda de mano de obra. Según este referente en la temática, el futuro apunta a la complementación antes que a la sustitución. Esto, a su vez, limitará la «polarización del empleo" que proponen otros autores, en tanto muchos puestos de trabajo de cualificación media seguirán exigiendo una mezcla de tareas de todo el espectro de cualificaciones (Autor, 2015, p. 27). En consonancia, según un estudio llevado a cabo por McKinsey (2016), aunque menos del 5\% de las ocupaciones pueden automatizarse por completo, alrededor del $60 \%$ tiene, al menos, un 30\% de actividades que pueden automatizarse.

Además, como subrayan Acemoglu y Restrepo (2016), la viabilidad técnica no siempre implica la viabilidad económica. Es decir, para que los empleos humanos sustituyan a las máquinas, el cambio debe ser comparativamente más rentable para las empresas que los anteriores procesos de producción con uso intensivo de mano de obra. En la misma línea, Eslake (2017) cita una serie de factores que influyen en la decisión de reemplazo de trabajadores por máquinas: grandes costos iniciales, riesgos legales y regulatorios, el sentimiento del cliente, entre otros. De hecho, McKinsey (2015) estima que la automatización podría reemplazar el 45\% de las actividades que actualmente realizan los seres humanos, pero que 
solo el $5 \%$ de los puestos de trabajo plenos podrían ser totalmente sustituidos por la tecnología. El informe «Dividendos digitales», publicado por el Banco Mundial en el 2016, comparte esta perspectiva, al entender que el proceso de automatización se moderará principalmente debido a los salarios más bajos y la mayor lentitud de adopción de las nuevas tecnologías en algunos países (p. 23).

Tras advertir estas limitaciones sobre las predicciones de Frey y Osborne, Arntz y otros (2016) utilizaron diferentes conjuntos de datos que examinaron el trabajo a nivel de tarea para modelar el impacto de la automatización en distintos países de la OCDE. Su modelización mostró que, para Estados Unidos, solo alrededor del 9\% de los puestos de trabajo estarían en riesgo. Se destacan en su trabajo, además del hincapié sobre las limitaciones (económicas, legales y sociales), la incorporación de tecnología y la creación de empleos derivados de las nuevas tecnologías y el aumento de productividad, la elevada heterogeneidad de riesgo de automatización entre los países, que oscila entre el $6 \%$ en Corea hasta el $12 \%$ en Austria.

Finalmente, diversos informes resaltan que, aunque el uso de varias tecnologías disruptivas en torno a la industria 4.0 ha aumentado, hasta ahora su despliegue parece estar concentrado en un pequeño número de países. Los robots industriales, por ejemplo, han experimentado una tasa de crecimiento anual del $17 \%$ desde 2010 , pero el $75 \%$ de ellos están siendo utilizados únicamente en cinco países. De hecho, una encuesta de ManpowerGroup (2017) a 18000 empleadores de 43 países señala que el $64 \%$ de ellos cree que la tecnología no tendrá ningún efecto en su plantilla en los próximos dos años. Por el contrario, el 19\% afirma que la automatización podría aumentar los puestos de trabajo de su empresa, y solo el $12 \%$ estima una disminución. Más concretamente, los países de Europa meridional y América Latina, junto con Estados Unidos, Reino Unido, Nueva Zelanda, Taiwán y Sudáfrica, tienden a predecir aumentos en sus tasas de empleo, mientras que la India y los empleadores de los países de Europa central y oriental tienen la intención de despedir a los trabajadores mediante la automatización. Además, la mitad de los ejecutivos encuestados también sospecha que la automatización estimula la creatividad, ya que libera a los empleados para hacer el trabajo que quieren hacer. De hecho, según un estudio de ServiceNow (2017), el 91\% de los encuestados confirmaron que sus empleados calificados dedican demasiado tiempo a tareas administrativas.

Tal como puede advertirse en esta breve reseña de los trabajos de mayor relevancia y resonancia dentro de la amplia literatura del futuro del trabajo, las aguas están divididas por fracturas prácticamente insalvables. Si bien pueden reconocerse ciertos consensos básicos al interior de los 
dos principales extremos del continuo (pesimistas y optimistas tecnológicos), la distancia entre sus argumentos -e incluso sus conceptos- y la diversidad de metodologías de cálculo de predicciones utilizadas y de regiones e industrias analizadas provoca que la literatura al respecto se encuentre lejos (aunque cada vez más cerca) de alcanzar bases sólidas de análisis que permitan profundizar sobre otro tipo de discusiones. En términos epistemológicos, la polarización de opiniones responde no tanto al tipo de metodología utilizada, sino más bien a las variables consideradas $y$, fundamentalmente, a la diferencia entre "posibilidad de automatización» frente a "probabilidad de automatización» de diferentes tipos de empleos. En la siguiente tabla se resumen los principales argumentos de estas posiciones.

TABLA 2. Resumen de principales argumentos sobre el futuro del empleo

\section{Pesimistas tecnológicos}

Las nuevas tecnologías permiten el reemplazo de los trabajadores con empleos que implican tareas principalmente repetitivas a costos reducidos.

\section{Optimistas tecnológicos}

El impacto de las nuevas tecnologías sobre el mercado laboral será relativamente menor; procesos similares han ocurrido en el pasado y el mercado laboral se ha expandido gracias a estos cambios.

Al impactar sobre todo tipo de industrias (tanto manuales como cognitivas), los cambios tecnológicos actuales tienen un menor potencial de creación de empleo que otras revoluciones del pasado.

Los requisitos de los nuevos puestos laborales más demandados requieren habilidades y conocimiento difíciles de adquirir.

Los cambios tecnológicos promueven la creación y el aumento de la demanda de nuevos puestos laborales anteriormente inexistentes.

La automatización de tareas no implica el reemplazo de puestos completos. Es más probable la complementariedad entre humanos y procesos automatizados, lo que aumentará la productividad de los primeros.

La regulación de mercados e instituciones que se requiere como respuesta a los cambios tecnológicos demorará mucho tiempo, lo que otorga ventajas a las nuevas tecnologías por sobre los trabajadores.

Las nuevas tecnologías continuarán reduciendo sus costos y aumentando sus impactos en términos de productividad hasta el punto de quebrar las barreras económicas e institucionales, incluso en los países más tecnológicamente atrasados, lo que resultará en adopciones tardías, pero más abruptas y con menor oportunidad de adaptación para los trabajadores.
Que un empleo sea factible de ser automatizado no significa que lo será. Hay diversas barreras de tipo económico e institucionales que limitan los incentivos al reemplazo de los trabajadores.

El despliegue de las tecnologías más disruptivas aún está concentrado en un número escaso de países e industrias. 


\section{El impacto sobre la desigualdad social}

Como da cuenta el debate mencionado, aún es imposible determinar con certeza si estas tecnologías destruirán más puestos de trabajo de los que crearán en el corto y mediano plazo. Por otra parte, si bien hay cierto consenso básico en que la difusión de estas permitirá generar mayor riqueza, esto no significa que esté necesariamente mejor distribuida. Existe un acuerdo generalizado acerca de que la tecnología ya ha causado y seguirá contribuyendo a la polarización de la estructura ocupacional. Aunque se crearán muchos nuevos puestos de trabajo, los más remunerados requerirán mayores niveles de formación para prosperar en un ambiente de trabajo más automatizado. El costo de este ajuste no se distribuirá equitativamente entre países, comunidades u ocupaciones. La transición será especialmente dolorosa para los que no estén preparados. Podemos hablar entonces de efectos contrapuestos en los tres niveles generales de capacitación y escala salarial:

1. En primer lugar, con la caída de los precios de la tecnología, las habilidades de resolución de problemas se vuelven más demandadas, lo que explica el crecimiento sustancial del empleo en ocupaciones que implican tareas cognitivas donde la mano de obra calificada tiene una ventaja comparativa, así como el aumento persistente en los retornos a la educación (Acemoglu, 2002; Autor y Dorn, 2013). En este sentido, hay evidencia bastante clara de que la innovación presenta un sesgo de habilidades, es decir, favorece la demanda de mano de obra altamente calificada (Aboal y Zunino, 2017).

2. Al mismo tiempo, es probable que aumente la demanda en ocupaciones no rutinarias de carácter manual, que hoy por hoy son difícilmente automatizables (por ejemplo, servicios personales, cierto tipo de servicios alimentarios, limpieza y seguridad), generalmente producidas por personas de bajo nivel educativo y con bajos salarios (Autor y Dorn, 2009; Autor y Dorn, 2013).

3. Finalmente, se estima que el gran descenso de la demanda se dará en ocupaciones de nivel medio, que en general requieren de actividades tanto de orden físico como cognitivo, pero caracterizadas principalmente por ser repetitivas y rutinarias. Los motivos son evidentes: muchos de esos puestos de calificación media siguen reglas bien definidas cada vez más sencillas de codificar y computarizar (Autor, 2017). Estas tareas comprenden tanto operarios de fábricas como trabajadores de cuello blanco, privados y estatales.

La consecuencia de la combinación de estos efectos implica que los mercados de trabajo se volverán cada vez más polarizados, con un aumento en la demanda de ocupaciones en extremos opuestos de la distribución salarial. Los economistas han descrito estos impactos como «el 
vaciado del medio» (Autor y Dorn, 2013; Autor, 2015), como polarización del empleo (Goos et al., 2014; Fernández-Macías y Hurley, 2016) o como «se acabó el promedio" (Cowen, 2013). Un amplio cuerpo de literatura confirma la presencia de polarización del empleo en distintas industrias, localidades y naciones (Autor, Katz y Kearney 2006, 2008; Goos y Manning, 2007; Michaels, Natraj y Van Reenen 2014; Goos, Graetz y Michaels, 2015; Autor, Dorn y Hanson, 2015).

Goos et al. (2014) muestran que la demanda de empleos medianamente remunerados disminuye en comparación con las ocupaciones con salarios altos y bajos porque, en primer lugar, el cambio tecnológico actual está sesgado hacia la sustitución de la mano de obra en tareas rutinarias $y$, en segundo lugar, porque existe el fenómeno de la deslocalización de tareas. Los autores encuentran que la polarización del empleo apareció en 16 países de Europa occidental y fue generalizada en las economías avanzadas en el periodo 1993-2010: mientras que las ocupaciones mejor remuneradas muestran los incrementos más rápidos en su participación en el empleo (por ejemplo, las de gerencia, profesionales y profesionales asociados), la participación de las que pagan alrededor de la mediana del salario ocupacional (oficinistas, trabajadores de artesanías y oficios relacionados, y operadores y ensambladores de plantas y máquinas) ha caído. El informe del Banco Mundial «Dividendos digitales» (2016, p. 22) llega a conclusiones similares en términos descriptivos de variación anual promedio sobre una base de datos de 35 países industrializados y su evolución entre 1995 y 2012. En 25 de ellos se generó una disminución de ocupaciones de calificación intermedia y un aumento de ocupaciones de baja calificación y, sobre todo, de alta calificación.

Esta dinámica tiene grandes implicaciones para la movilidad social y la sostenibilidad de las clases medias. También pone de manifiesto la importancia de la educación y la formación profesional, es decir, la inversión en el capital humano como activo clave en la era de la economía del conocimiento (Salazar-Xirinachs, 2017). Berriman (2017) encuentra, por ejemplo, que para los trabajadores individuales el factor clave de diferenciación al momento de calcular su probabilidad de reemplazo es la educación. En un estudio sobre el Reino Unido, calcula que el riesgo potencial estimado de automatización y reemplazo varía entre el $12 \%$ y el $46 \%$ (casi cuatro veces más), según los niveles de educación alcanzados. Como advierte Galiani (2017): “Hoy más que nunca, tenemos que trabajar sobre las calificaciones de la población. La educación también tiene que cambiar. El foco debe estar en enseñar a aprender".

Además, es primordial resaltar que los países en desarrollo con una ventaja competitiva de mano de obra barata se enfrentan tal vez a los 
riesgos más graves, ya que las tecnologías emergentes podrían llevar a la reubicación de la manufactura a los países de altos ingresos (WEF, 2016). En América Latina, parte sustancial de las exportaciones y del empleo se concentra en actividades que corren un alto riesgo de ser automatizadas, como la manufactura intensiva en mano de obra, la extracción de recursos naturales y los servicios de calificación media, como los contables, legales o de gestión. Frente a esto, un futuro mejor depende de que se pongan en práctica políticas de desarrollo productivo, innovación y talento humano que aceleren el crecimiento de la productividad, y de un patrón de crecimiento más inclusivo y sostenible (Salazar-Xirinachs, 2017). «Para facilitar esta transición y mitigar sus efectos sociales adversos, la educación, la capacitación permanente y una adecuada red de contención, además de un apropiado sistema de incentivos impositivos, ocuparán un rol central»» (De Zavalia et al, 2019).

Tal como es esperable, el debate sobre la cohesión social también ha sido abordado por diversas organizaciones sociales, como sindicatos, confederaciones y gremios. Sin embargo, en lo que a estas instituciones concierne, no existe un consenso generalizado en cuanto a los instrumentos a implementar para garantizar una transición netamente positiva hacia la digitalización. Frente a esto, un informe publicado por la Organización Internacional del Trabajo, que sintetiza una ronda de diálogos nacionales llevados a cabo por 168 Estados miembros y en la que participaron gobiernos, organizaciones de trabajadores y empleadores, círculos académicos y otros miembros de la sociedad, enfatiza la importancia del diálogo social como instancia para encontrar soluciones, generar confianza y mejorar las capacidades de negociación (2017, p. 70).

En este sentido, la discusión sobre el devenir del trabajo fue abordada principalmente por organizaciones sociales de países desarrollados que ya se encuentran viviendo parte de los cambios que traen los avances tecnológicos en el sector laboral como Estados Unidos, Reino Unido y países de la Unión Europea; mientras que en los países menos desarrollados, particularmente en la zona de América Latina, solo se han producido encuentros informales y meramente enunciativos sobre los desafíos que representa la cuarta Revolución Industrial, dado que aún al día de hoy continúa siendo un tema apenas estudiado en la región (Bitar, 2019).

Entre las organizaciones sociales que han llevado a cabo estudios relativos al futuro del trabajo se destacan principalmente cuatro: AFL-CIO (American Federation of Labor and Congress of Industrial Organizations) de Estados Unidos y Canadá, TUC (Trade Union Congress) de Reino Unido y CCOO (Comisiones Obreras), y UGT (Unión General de Trabajadores) de España. En los informes publicados por tales entidades se subraya el 
factor de la incertidumbre como condicionante para identificar la cantidad de empleos ganados o perdidos, tomando varios de los estudios desarrollados anteriormente en este trabajo. En el informe publicado por CCOO/UGT, se definen los problemas existentes en la actualidad como aspectos por atender de manera urgente para garantizar un "trabajo decente" (OIT, 2017) en el futuro, entre ellos la precariedad laboral, la informalidad en el empleo, la caída percibida en los salarios, entre otros (CCOO y UGT, 2017, p. 3). Por otro lado, tanto los informes de AFL-CIO como de TUC desarrollan los principales tópicos que serían problemáticos a futuro: la carga horaria, el salario justo, el uso de big data como forma de llevar un control de los trabajadores, el aprendizaje y la capacitación continuos, entre otros (AFL-CIO, 2019; TUC, 2018). Sin embargo, todos estos estudios concuerdan en la necesidad de fomentar la negociación colectiva (o collective bargaining) como forma de garantizar una transición en la que se asegure a los trabajadores buenas condiciones laborales (AFL-CIO, 2019). Por otra parte, más allá de recomendaciones enunciativas para mitigar los impactos de estos fenómenos, ninguno aborda de manera específica la pregunta planteada por este trabajo, en términos de predicciones concretas sobre el número de puestos de trabajo en el futuro.

\section{Conclusiones}

Como pudo observarse, los analistas están lejos de ponerse de acuerdo sobre el futuro del trabajo. Las predicciones van de optimistas a devastadoras, y difieren en decenas de millones de empleos, incluso cuando se comparan plazos similares. La amplia gama de proyecciones se deriva de la incertidumbre sobre cuántos empleos se eliminarán totalmente, cuántos se adaptarán y cuántos se crearán con el surgimiento de nuevos sectores. Más aún, esta amplia diferencia en términos de predicciones parte de diversas variaciones en términos metodológicos (fundamentalmente, aquella que enfrenta el enfoque por puestos del enfoque por tareas), de región y de industria analizada. Asimismo, diversas predicciones se estructuran únicamente como el resultado de una sola tecnología, como los vehículos autónomos o los robots industriales, mientras que otras pretenden una mirada más abarcadora e integral. Finalmente, parte importante de la diferenciación en términos cuantitativos sobre los distintos resultados se estructura a partir de la discrepancia de miradas epistemológicas entre la posibilidad y la probabilidad de pérdida de empleos. El argumento más comúnmente presentado es aquel que incurre en que «la posibilidad de que un trabajo sea automatizado no significa que sea eficiente hacerlo".

Las cifras más comúnmente citadas provienen de tres lugares: el famoso estudio de Frey y Osborne (2013) que dice que el $47 \%$ de los empleos en Estados Unidos. están en alto riesgo de automatización en las próximas 
décadas, un estudio de la OCDE que sugiere que el $9 \%$ de los empleos en los 21 países miembros de la organización son automatizables, y un informe de McKinsey G. C. de 2018 que dice que entre 400 y 800 millones de empleos en todo el mundo podrían automatizarse para 2030.

\section{Áreas de controversia en la literatura:}

- El alcance y la velocidad del impacto de las nuevas tecnologías en el número de puestos laborales.

- El alcance del impacto en cuanto a su homogeneidad o heterogeneidad a lo largo de distintas industrias y países.

- Algunos autores sostienen que la velocidad del cambio es más rápida que nunca, mientras que otros no están de acuerdo.

En resumen, aunque estas predicciones son hechas por docenas de expertos mundiales en economía y tecnología, nadie parece estar en la misma página. En realidad, solo hay una conclusión significativa: no tenemos ni idea de cuántos puestos de trabajo se perderán o ganarán realmente con el avance del progreso tecnológico. Sin embargo, también es posible advertir otras áreas de un creciente consenso entre los analistas.

\section{Áreas de consenso en la literatura:}

- El impacto en el empleo se producirá, en gran medida, a nivel de tareas antes que de reemplazo de puestos completos (mayor consenso sobre esta metodología de cálculo en los últimos años).

- Si bien existe la posibilidad de que algunos empleos desaparezcan, es más probable que cambien; algunos de manera tan sustancial que ya no serán reconocibles y otros de manera mínima.

- Al mismo tiempo, se crearán nuevos puestos de trabajo que pueden requerir nuevas cualificaciones que aún no se han identificado.

- Es probable que se desarrolle un fenómeno de «vaciamiento del medio» en términos sociales, en tanto que las ocupaciones con menor riesgo de automatización se encuentran en los extremos opuestos de las escalas de ingresos y de requisitos educativos y cognitivos.

- La participación en el aprendizaje permanente será clave a medida que la tecnología cambie los puestos de trabajo y los lugares de trabajo. Los trabajadores tendrán que volver a desarrollar sus habilidades y atributos a lo largo de su vida para permanecer en la fuerza laboral.

En términos de limitaciones, advertimos que la literatura aún no ha consensuado en términos metodológicos sobre la mejor forma de medir la probabilidad de automatización, aunque hay una tendencia creciente en el consenso a utilizar el «enfoque por tareas» antes que el «enfoque por puestos», lo que ha dado por resultado una superación de las visiones 
más pesimistas sobre la pérdida de puestos de trabajo. Asimismo, si bien se han desarrollado importantes contribuciones en términos de cálculos estadísticos sobre diversas bases de datos, revisiones históricas, entrevistas y encuestas en los niveles nacional y regional, un enorme porcentaje de la literatura acude únicamente a la reflexión abstracta, sin estudios empíricos concretos que sustenten las conclusiones abordadas. Si bien la proyección hacia el futuro en términos cuantitativos (más allá de la metodología utilizada para la obtención y análisis de datos) sigue siendo un acto especulativo, permite discusiones más claras, además de mayor facilidad de sistematización de la literatura y estructuración de categorías, que la opinión teórica sin fundamento empírico que caracteriza la mayor parte de los trabajos analizados. Igualmente, la falta de consensos metodológicos sobre los citados trabajos empíricos ha tendido a disminuir su impacto en términos científicos, a pesar de que varios de estos trabajos han logrado importante influencia a nivel político y social.

Cabe resaltar, sin embargo, la amplitud geográfica de los estudios encontrados, que cubren prácticamente todo el mundo. Si bien hay una mayor concentración de estudios sobre los impactos en los países desarrollados (principalmente, países de la OCDE), parte de la bibliografía también aborda el impacto sobre países en vías de desarrollo, a nivel general e incluso en regiones y casos específicos. Empero, cabe mencionar que no hemos encontrado (suponemos que principalmente a causa de nuestras barreras idiomáticas antes que por la falta real de fuentes), estudios en profundidad sobre los casos de India o China, en los que, presumimos, el impacto de los procesos estudiados será significativo.

Se destaca también la amplitud en términos de los sectores económicos abordados. Hemos encontrado estudios que incluyen industrias tan diversas como la manufactura, el agro, la administración pública, la logísti$\mathrm{ca}$, la gastronomía, el periodismo, la medicina e incluso el arte, entre otras. Se proyectan cambios importantes en prácticamente todas las ramas de la actividad económica. Mientras que algunas profesiones desaparecerán por completo y otras nuevas se crearán, la mayor parte experimentará cambios radicales en términos de la complementación entre los seres humanos y los procesos automatizados.

Además, cabe resaltar que varios de estos procesos se han comenzado a acelerar ante los cambios generados por la pandemia de COVID-19 desatada a nivel mundial desde principios de año, que han despertado la curiosidad de las empresas y otras instituciones por los procesos automatizados y las tareas a distancia. Si bien este fenómeno se sopesa frente a la crisis económica internacional aparejada, que dificulta afrontar los costos necesarios para adquirir algunos de estos insumos, es esperable observar 
nuevas y fugaces adaptaciones a medida que las empresas descubran las ventajas (y desventajas) de estas nuevas tecnologías, con resultados aún difíciles de calcular. Los impactos de largo plazo todavía no son claros. Frente a estos cambios, el futuro del trabajo es hoy más incierto que nunca. Priorizar una educación que permita afrontar este futuro, por el contrario, es cada vez más importante.

\section{Contribución de los autores}

Lautaro Rubbi es director de la investigación y autor principal del artículo. Bruna Barlaro Rovati es autora secundaria del texto y responsable de la búsqueda bibliográfica y del análisis de datos. Axel Petraglia es autora terciaria y responsable de la búsqueda bibliográfica.

\section{Fuente de financiamiento}

UADE.

\section{Conflicto de interés}

Los autores declaran no tener compromisos de interés. 


\section{REFERENCIAS BIBLIOGRÁFICAS}

Aboal, D. y Zunino, G. (2017). Innovaciones y habilidades en América Latina. En: INTAL (2017). Robotlución. INTAL. Integración \& Comercio. 40. Recuperado de https://publications.iadb.org.

Acemoglu, D. (2002). Technical change, inequality, and the labor market. Journal of Economic Literature, 40, pp. 7-72.

Acemoglu, D. y Restrepo, P. (2016). The race between machine and man: Implications of technology for growth, factor shares and employment. NBER Working Paper 22252.

Acemoglu, D. y Restrepo, P. (2017). Robots and jobs: evidence Form US and the labour markets. NBER Working Paper 23285.

AFL-CIO (2019). The future of work and unions. AFL-CIO Commission. Recuperado de https://aflcio.org/reports/afl-cio-commission-future-workand-unions

Apella, I. y Zunino, G. (2017). Cambio tecnológico y el mercado de trabajo en Argentina y Uruguay: un análisis desde el enfoque de tareas. Informe Técnico del Banco Mundial 11.

Arntz, M., Gregory, T. y Zierahn, U. (2016). The risk of automation for jobs in $O E C D$ countries, OECD social, employment and migration. Working Papers, 189. París: OECD Publishing.

Atkinson, R. y Brown, J. (2017). The future of work, a guide for transatlantic policymakers. Information Technology \& Innovation Fundation. Recuperado de https://itif.org/publications/2018/12/17/future-work-guide-transatlantic-policymakers

Atkinson, R. y Wu, J. (2017). False alarmism: technological disruption and the U.S. labor market, 1850-2015. Information Technology \& Innovation Fundation.

Autor, D. (2015). Why are there still so many jobs? The history and future of workplace automation. Journal of Economic Perspectives, 29(3), pp. 3-30.

Autor, D. (2016). Automatización y empleo: de qué deberíamos preocuparnos (y de qué no). Boletín Informativo Techint 354.

Autor, D. H., Levy F. y Murnane R. J. (2003). The skill content of recent technological change: an empirical exploration. The Quarterly Journal of Economics, 118(4), pp. 1279-1333.

Autor, D. H. y Dorn, D. (2013). The growth of low-skill service jobs and the polarization of the US labor market. American Economic Review, 103(5), pp. 1553-1597. 
Autor, D. H., Dorn D, y Hanson, G. H. (2015). Untangling trade and technology: evidence from local labor markets. Economic Journal, 125(584), pp. 621-646.

Autor, D. H., Katz, L. y Kearney, M. (2008) Trends in US wage inequality: Revising the revisionists. The Review of Economics and Statistics, 90(2), pp. 300-323.

Baert, A. y Ledent, P. (2015). La révolution technologique en Belgique. ING Focus-Emploi. Bruselas: ING.

Bakhshi, H., Downing, J. M., Osborne, M. A., y Schneider, P. (2017). The future of skills: employment 2030. Londres: Pearson and Nesta.

Banco Mundial (2016). World Development Report 2016: Digital Dividends. Washington: The World Bank.

Beliz, G. (2017). Un contrato social tecnológico para América Latina. En Robotlución. INTAL. 2017. Integración \& Comercio. 40. Recuperado de https://publications.iadb.org

Berriman, R. (2017). Will robots steal our jobs? The potential impact of automation on the UK and other major economies. PricewaterhouseCoopers.

Bessen, J., Goos, M., Salomons, A. y Van Den Berge, W. (2019). Automatic Reaction: What happens to workers at firms that automate? CBS Working Paper.

Bowles, J. (2014). The computerisation of European jobs. Bruegel Blog. Recuperado de http://bruegel.org/2014/07/the-computerisation-of-european-jobs/

Brynjolfsson, E. y A. McAfee. (2011). Race against the machines: how the digital revolution is accelerating innovation, driving productivity, and irreversibly transforming employment and the economy. Massachusetts: Digital Frontier Press.

Brynjolfsson, E. y A. McAfee. (2014). The second machine age: work, progress, and prosperity in a time. Nueva York: Norton \& Company.

Carlson, M. (2015). The robotic reporter. Routledge, 3(3), pp. 416-431.

CBInsights (2017). The state of automation. Recuperado de https://www. cbinsights.com/research/report/state-of-automation/

CCOO y UGT (2017). El futuro del trabajo que queremos. Recuperado de http://docpublicos.ccoo.es/cendoc/049597FuturoTrabajoQueremos.pdf

Chui, M., Manyika, J. y Miremadi, M. (2019). Where machines could replace humans-and where they can't (yet). McKinsey Digital. Recuperado de https://www.mckinsey.com/business-functions/mckinsey-digital/ourinsights/where-machines-could-replace-humans-and-where-they-cantyet\# 
Cowen, T. (2013). Average is over: powering America beyond the age of the great stagnation. Nueva York: Dutton.

Cronin, P., Ryan, F. y Coughlan, M. (2008). Undertaking a literature review: A step-by-step approach. British journal of nursing (Mark Allen Publishing), 17(1), pp. 38-43.

De Zavalia, E., Del Águila, J., Cuesta, A. y Rubbi, L. (2019). Empresas, empleo y educación ante el cambio tecnológico. En Academias, Conocimiento y Sociedad (pp. 79-102). Buenos Aires: Academia Nacional de Ciencias Morales y Políticas.

DeCanio, S. J. (2016). Robots and humans - complements or substitutes? Journal of Macroeconomics, 49, pp. 280-291.

Degryse, C. (2016). Digitalisation of the economy and its impact on labour markets. ETUI Research Paper: Working Paper 2016.02.

Deloitte (2014). De Impact van Automatisering Op de Nederlandse Arbeidsmarkt.

Deloitte (2015). From brawn to brains. The impact of technology on jobs in the UK.

Deloitte. (2016). Transforming the Swiss economy: The impact of automation on employment and Industries. Recuperado de https://www2.deloitte. com/content/dam/Deloitte/ch/Documents/innovation/ch-en-cons-innovation-transforming-swiss-economy.pdf

Downey, M. (2016). Partial automation: Routine-biased technical change, deskilling, and the minimum wage. San Diego: University of California. Recuperado de http://econweb.ucsd.edu/ pmdowney/pdfs/wp/ PartialAutomation.pdf

Edmonds, D. y Bradley, T. (2015). Mechanical boon: will automation advance Australia? Office of the Chief Economist. Department of Industry, Innovation and Science.

Engler, J., Pritzker, P., Alden, E. y Taylor-Kale, L. (2018). The work ahead: machines, skills, and U.S. leadership in the twenty-first century. Council of Foreign Relations.

Eslake, S. (2017). Insecurity, inequality, and the labour market. Jobs Australia Conference. Hobart: ResearchGate.

Estevadeordal (2017). Estar preparados. En INTAL (2017). Robotlución. Integración \& Comercio. 40. Recuperado de https://publications.iadb.org

European Parliamentary Technology Assessment. (2016). The Future of labour in the digital era. ubiquitous computing, virtual platforms, and realtime production. Recuperado de http://epub.oeaw.ac.at/ita/ita-projektberichte/ EPTA-2016-Digital-Labour.pdf 
Fernández-Macías, E. y Hurley, J. (2016). Routine-biased technical change and job polarization in Europe. Socio-Economic Review, 15(3), pp. 563-585.

Finextra (2016). Banking on Blockchain: Charting the Progress of Distributed Ledger Technology in Financial Services. Finextra White Paper.

FIR (2016). World Robotics: Industrial Robots. Federación Internacional de Robótica (IFR).

FIR (2019). World Robotics 2019: Industrial Robots. Federación Internacional de Robótica (IFR).

Frey, C. B. y Osborne, M. A. (2013). The future of employment: how susceptible are jobs to computerisation? Technological Forecasting and Social Change, 114, pp. 254-280.

Fundación Bertelsmann (2015). 2050: The future of work. Recuperado de https://www.bertelsmann-stiftung.de/fileadmin/files/BSt/Publikationen/ GrauePublikationen/BST_Delphi_E_03lay.pdf

Galiani, S. (2017). Terminator IV. Boletín Techint.

German Federal Ministry of Labour and Social Affairs (2016). White PaperWork 4.0. Federal Ministry of Labour and Social Affairs.

Germany Trade and Invest (2018). The robotics \& automation industry in Germany. Federal Ministry for Economic Affairs and Energy of Germany. Recuperado de https://www.gtai.de/gtai-en/invest/service/publications/ the-robotics-automation-industry-in-germany-64312

Goldin, I. (2016). How immigration has changed the world -for the better. Geneva: World Economic Forum. Recuperado de https://www.weforum. org/agenda/2016/01/how-immigration-has-changed-the-world-for-thebetter/

Goos, M. et al. (2015). Employment growth in Europe: The role of innovation, local job multipliers. SSRN Electronic Journal.

Goos, M., Manning, A. y Salomons, A. (2009). Job polarization in Europe. The American Economic Review, 99(2), pp. 58-63.

Goos, M., Manning, A. y Salomons, A. (2014). Explaining job polarization: routine-biased technological change and offshoring. The American Economic Review, 104(8), pp. 2509-2526.

ILO. (2016). Non-standard employment around the world: understanding challenges, shaping prospects. Ginebra: Organización Internacional del Trabajo.

Laviña Orueta, J., Serrano, G. L. y Varela Ferrio, J. (2019). Innovación tecnológica y empleo. Foro Empresas Innovadoras. Recuperado de http:// foroempresasinnovadoras.com/wp-content/uploads/2019/11/Libro-OK_ v6-final.pdf 
Luksha, P. et al. (2015). Atlas of emerging jobs. Moscú: Skolkovo.

ManpowerGroup (2017). The skills revolution: digitalization and why skills and talent matter.

Manyika, J., Chui, M., Miremadi, M., Bughin, J., George, K., Willmott, P. y Dewhurst, M. (2017). A future that works: automation, employment, and productivity. McKinsey Global Institute.

McKinsey \& Company. (2015). The four global forces breaking all the trends. Nueva York.

McKinsey \& Company; alphaßeta; Macquarie Group Foundation (2018). A demand driven approach to addressing youth unemployment in Australia. Sydney: McKinsey \& Company; alphaßeta; Macquarie Group Foundation.

McKinsey and Company (2017). Jobs lost, jobs gained: What the future of work will mean for jobs, skills, and wages. Recuperado de https://www. mckinsey.com/featured-insights/future-of-work/jobs-lost-jobs-gainedwhat-the-future-of-work-will-mean-for-jobs-skills-and-wages

MGI (2013). Disruptive technologies: Advances that will transform life, business, and the global economy. Tech. Rep., McKinsey Global Institute.

Michaels, G., Ashwini, N. y Van Reenen, J. (2014). Has ICT polarized skill demand? Evidence from eleven countries over twenty-five years. Review of Economics and Statistics, 96(1), pp. 60-77.

Moretti, E. (2010). Local multipliers. American Economic Review, 100(2), pp. 373-377.

OCDE (2017). Computers and the future skills of job demand 2017. Recuperado de https://read.oecd-ilibrary.org/education/computers-and-thefuture-of-skill-demand_9789264284395-en\#page1

Organización Internacional del Trabajo (2017). ILO Glossary of Statistical Terms. Organización Internacional del Trabajo. Recuperado de https:// www.ilo.org/ilostat-files/Documents/Statistical\%20Glossary.pdf

Organización Internacional del Trabajo (2017). Síntesis de los diálogos nacionales sobre el futuro del trabajo. Recuperado de https://www.ilo.org/ wcmsp5/groups/public/---dgreports/---cabinet/documents/publication/ wcms_591507.pdf

Oxford Martin School (2015). Industrial Renewal in the 21st Century: Evidence from US Cities? Regional Studies, 51(3), pp. 404-413.

Pollin, R., Garrett-Peltier, H., Heintz, J. y Hendricks, B. (2014). Green Growth: A U.S Program for Controlling Climate Change and Expanding Job Opportunities. Center for American Progress. 
PricewaterhouseCoopers (2017). Will robots steal our jobs? The potential impact of automation on the UK and other major economies. PricewaterhouseCoopers.

Rofman, R. y Apella, I. (2017). Envejecimiento poblacional, cambio tecnológico y mercado de trabajo en Argentina. Boletín Techint.

RolandBerger (2014). Think act - Les classes moyennes face à la transformation digitale. RolandBerger.

Roos, G. y Shroff, Z. (2017). What will happen to the jobs? Technologyenabled productivity improvement - good for some, bad for others.

Salazar-Xirinachs, J. M. (2017). The future of work, employment, and skills in latin america and the caribbean. Organización Internacional del Trabajo. Recuperado de http://www.ilo.org/wcmsp5/groups/public/---americas/--ro-lima/---sro-port_of_spain/documents/publication/wcms_544337.pdf

Santanatoglia, E., Sosa Valle, F., Mariani, K. y Pivetta, G. (2018). Future of work and education for the digital age: providing a broader framework to social and labour relations. Think 20.

Schumpeter, J. (1942). Creative destruction. Capitalism, socialism and democracy, 825, pp. 82-85.

Schwab, K. (2016). The Fourth Industrial Revolution. World Economic Forum.

ServiceNow (2017). Today's State of Work: At the Breaking Point.

Smith, A. y Anderson, J. (2014). Al, robotics, and the future of jobs. Recuperado de http://www.pewinternet.org/2014/08/06/future-of-jobs/

The Economist (2018). The Automation readiness index. Recuperado de http://www.automationreadiness.eiu.com/

The Economist (2018). The Automation readiness index. Recuperado de http://www.automationreadiness.eiu.com/

Torres Muñoz, R. (2019). Políticas públicas para la robótica y la inteligencia artificial. Biblioteca del Congreso Nacional de Chile. Recuperado de https://www.bcn.cl/obtienearchivo?id=repositorio/10221/26982/1/Politicas_Publicas_para_la_Robotica_y_la_Inteligencia_Artificial.pdf

TUC (2018). A future that works for working people. Recuperado de: https:// www.tuc.org.uk/research-analysis/reports/future-works-working-people

Valenduc, G. y Vendramin, P. (2017). Digitalisation, between disruption and evolution. European Review of Labour and Research, 23(2), pp. 121-134.

WEF (2018). Informe sobre la preparación para el futuro de la producción 2018. Foro Económico Mundial 2018. Recuperado de http://www3.weforum.org/docs/FOP_Readiness_Report_2018.pdf 
West, D.M. (2015). What Happens If Robots Take the Jobs? The Impact of Emerging Technologies on Employment and Public Policy. Center for Technology Innovation at Brookings.

World Economic Forum (2016). The Fourth Industrial Revolution: what it means, how to respond. Recuperado de https://www.weforum.org/agen$\mathrm{da} / 2016 / 01 /$ the-fourth-industrial-revolution-what-it-means-and-how-torespond/

World Economic Forum. (2016). Values and the Fourth Industrial Revolution: Connecting the Dots Between Value, Values, Profit and Purpose. Global Agenda Council (2014-2016). Cologny: World Economic Forum.

World Economic Forum. (2017b). Robot revolution: the economics of automation.

alphaßeta (2017). The Automation Advantage. Sydney: alphaßeta. Recuperado de https://www.alphabeta.com/wp-content/uploads/2017/08/ The-Automation-Advantage.pdf 